\title{
Sustainable use of renewable resources in a stylized social-ecological network model under heterogeneous resource distribution
}

\author{
Wolfram Barfuss $^{1,3}$, Jonathan F. Donges ${ }^{1,4}$, Marc Wiedermann ${ }^{2,3}$, and Wolfgang Lucht ${ }^{1,5,6}$ \\ ${ }^{1}$ Earth System Analysis, Potsdam Institute for Climate Impact Research, Telegrafenberg A31, 14473 Potsdam, \\ Germany \\ ${ }^{2}$ Transdisciplinary Concepts \& Methods, Potsdam Institute for Climate Impact Research, Telegrafenberg A31, \\ 14473 Potsdam, Germany \\ ${ }^{3}$ Department of Physics, Humboldt University, Newtonstraße 15, 12489 Berlin, Germany \\ ${ }^{4}$ Stockholm Resilience Centre, Stockholm University, Kräftriket 2B, 11419 Stockholm, Sweden \\ ${ }^{5}$ Department of Geography, Humboldt University, Unter den Linden 6, 10099 Berlin, Germany \\ ${ }^{6}$ Integrative Research Institute on Transformations of Human-Environment Systems, Humboldt University, \\ Unter den Linden 6, 10099 Berlin, Germany \\ Correspondence to: Wolfram Barfuss (barfuss@ pik-potsdam.de)
}

Received: 13 April 2016 - Discussion started: 30 May 2016

Revised: 3 February 2017 - Accepted: 24 February 2017 - Published: 11 April 2017

\begin{abstract}
Human societies depend on the resources ecosystems provide. Particularly since the last century, human activities have transformed the relationship between nature and society at a global scale. We study this coevolutionary relationship by utilizing a stylized model of private resource use and social learning on an adaptive network. The latter process is based on two social key dynamics beyond economic paradigms: boundedly rational imitation of resource use strategies and homophily in the formation of social network ties. The private and logistically growing resources are harvested with either a sustainable (small) or non-sustainable (large) effort. We show that these social processes can have a profound influence on the environmental state, such as determining whether the private renewable resources collapse from overuse or not. Additionally, we demonstrate that heterogeneously distributed regional resource capacities shift the critical social parameters where this resource extraction system collapses. We make these points to argue that, in more advanced coevolutionary models of the planetary social-ecological system, such socio-cultural phenomena as well as regional resource heterogeneities should receive attention in addition to the processes represented in established Earth system and integrated assessment models.
\end{abstract}

\section{Resource use in social-ecological systems}

Whether, when and how human usage of biophysical resources meets limits that produce feedbacks onto social functioning has a long history of controversial discussion (Malthus, 1798; Meadows et al., 1972; Rockström et al., 2009). Especially in the last century, human activities have changed the relationship between nature and society at the global scale (Crutzen, 2002; Steffen et al., 2007, 2015a), making them mutually interdependent in an unprecedented manner and the question of their joint dynamics urgent. Social and ecological systems should therefore be studied not only in isolation but also as interlinked social-ecological systems (Berkes and Folke, 1998). Here, we contribute to this debate by investigating properties of a stylized social system that cause the linked resource use system to either collapse or remain viable. Such a perspective also has important implications for the mathematical modeling of interdependent, global human-environment interactions (Verburg et al., 2016; van Vuuren et al., 2016). Typically, in present- 
day analysis the Earth system is either modeled from a purely biophysical point of view (Claussen et al., 2002) or from a biophysical-economic one (van Vuuren et al., 2012), depending on the scope of the research question. However, both approaches do not take into account social dynamics beyond macroeconomic paradigms.

Here, we conceptually explore avenues for a third strand of global modeling, next to the biophysical and biophysicaleconomic one, also incorporating socio-cultural dynamics. Founded on a genuinely social-ecological perspective, we term these "World-Earth" system models to emphasize the free coevolution of the social and ecological components (Schellnhuber, 1998, 1999). While sophisticated models of this type are not yet available, the literature contains various modeling studies that incorporate potentially important features such as static interaction networks (Chung et al., 2013; Sugiarto et al., 2015) to depict stylized social dynamics (Holme and Newman, 2006; Auer et al., 2015), tele-coupling effects in a globalized society interacting through social networks (Janssen et al., 2006; Bodin and Tengö, 2012), socialecological regime shifts (Scheffer et al., 2001; Lade et al., 2013) and (social) tipping elements (Schellnhuber, 2009; Bentley et al., 2014), structural reorganization occurring on adaptive social networks (Gross and Blasius, 2008; Snijders et al., 2010; Sayama et al., 2013; Schleussner et al., 2016) or structural transformations (Lade et al., 2017) and cultural preference dynamics due to traits such as imitation (Traulsen et al., 2010) or homophily (McPherson et al., 2001; Centola et al., 2007).

We set out a simple model (see Sect. 2) to demonstrate that social network interactions, imitation and homophily may have a profound influence on the environmental state, such as determining whether a collection of private renewable resources collapses from overuse or not. We argue that more elaborate and sophisticated implementations of such social phenomena should receive attention in the future development of global system models, supplementing already established Earth system and integrated assessment models, neither of which at present include them.

As a particular case study for our model we examine the effect of heterogeneously distributed resources. This is important since in the real-world agents do have access to different amounts of biophysical resources. Our study examines under which combinations of parameters characterizing a social learning network process does the model converge to a sustainable regime for different degrees of resource access heterogeneity. Parameters governing social learning dynamics are, on the one hand, a homophily parameter $\phi$, addressing the propensity of nodes to establish interactions with nodes of the same kind (see Sect. 2 for a detailed model description). On the other hand, the timescale of social interaction $\tau$ quantifies the average time for social updates on the network. We purposely do not model any form of individual learning of the agents with regard to the best harvesting strategy to emphasize the effects of the described social learning process. For homogeneous resource access (Wiedermann et al., 2015), one already observes a threshold in the parameter space of the model from non-sustainable to sustainable regimes at certain critical values $\phi_{\mathrm{c}}$ and $\tau_{\mathrm{c}}$. Since the concrete heterogeneous resource distribution is often unknown, we show systematically how an increasing heterogeneity - starting from an almost homogeneous distribution - affects the critical transition parameters $\phi_{\mathrm{c}}$ and $\tau_{\mathrm{c}}$. Additionally we show that in our stylized model a heavy-tailed resource distribution in comparison to a non-heavy-tailed distribution changes the model's behavior considerably. This is important as real-world resource data suggest that access to biophysical resources may indeed be distributed with heavy tails.

\section{Model description}

The intention behind our model design is not to closely follow any specific real-world setting but to explore the coevolution of socio-cultural dynamics with ecological dynamics. On a conceptual level, human-environment interactions are happening either in a common-pool or private-pool setting. Common-pool dilemmas have been studied extensively in the past (Hardin, 1968; Tavoni et al., 2012; Ostrom, 2015). Here, agents can retrieve information on another agent's harvesting strategy either via the ecological subsystem, i.e., the common pool, itself or via purely social interactions. In order to specifically focus on the latter of the two as an important domain of processes, we eliminate any transfer of information via the ecological system and discard a common-pool setting in favor of individual and private resource stocks per agent. Wiedermann et al. (2015) introduced a model for such a setting for the special case of homogeneously distributed private resources, revealed transitions and distinct regimes in its parameter space, and provided analytical approximations of its dynamics. Here, we adjust this setting for the more general case of an inhomogeneous resource distribution. An overview of the model is provided in Fig. 1.

\subsection{A stylized anthroposphere}

The social learning (Bandura, 1977) process takes place in a network initialized as a random graph $G$ (Erdös and Rényi, 1960 ) with nodes labeled by integer number $i=1, \ldots, N$ that represent social agents. It is based on two theoretical paradigms: (i) agents either change their strategy through boundedly rational imitation (Traulsen et al., 2010; Bahar et al., 2014) or (ii) adapt their local network structure by rewiring to other nodes with similar behavior (homophily, McPherson et al., 2001; Centola et al., 2007). In order to integrate this discrete update process (Holme and Newman, 2006; Zanette and Gil, 2006) with the continuous evolution of the resource stocks, social update times $t_{i}$ are assigned to the agents as generated by a Poisson process with an expo- 


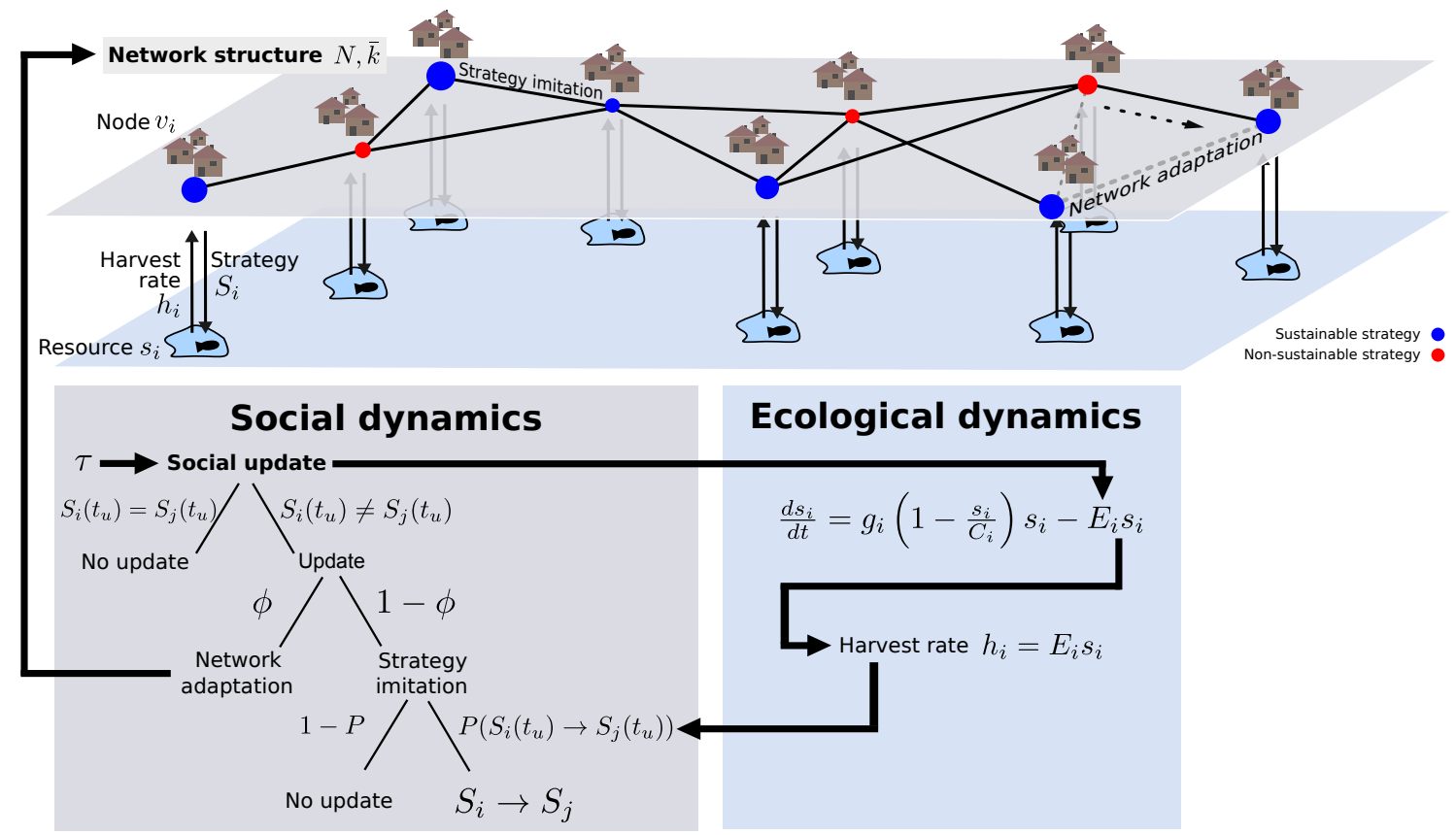

Figure 1. Illustration of our stylized social-ecological model. As the ecological subprocess the agents harvest their private logistically growing renewable resource with either a sustainable (blue) or non-sustainable (red) strategy. The social subprocess follows the logics of strategy imitation due to comparisons of harvest rates and of social network adaptation due to homophily. The social update times are generated by a Poisson process with average inter-event time $\tau$.

nential distribution,

$p\left(\Delta t_{i} ; \tau\right)=\frac{1}{\tau} \exp \left(\frac{-\Delta t_{i}}{\tau}\right)$,

of waiting times $\Delta t_{i}$, where the parameter $\tau$ gives the expected waiting time.

Thus, agent $v_{i}$ with the lowest update time in the queue performs the social update process accordingly:

- (1) If the degree of agent $v_{i}$ is zero (i.e., $v_{i}$ has no neighbors), move to (3); otherwise choose a neighbor $v_{j}$ of $v_{i}$ at random.

- (2) If $v_{j}$ and $v_{i}$ employ the same harvesting strategy $S_{i}=S_{j}$ (either sustainable or non-sustainable; see below), move to (3). Otherwise, move to (2.1).

- (2.1) With rewiring probability $\phi$ disconnect $v_{j}$ from $v_{i}$ and connect $v_{i}$ to a randomly chosen agent $v_{k}$ that employs the same strategy.

- (2.2) If (2.1) was not chosen, change the strategy of $v_{i}$ to the one of $v_{j}$ according to the sigmoidal imitation probability function

$$
P\left(S_{i} \rightarrow S_{j}\right)=\frac{1}{2}\left(\tanh \left(\gamma\left[h_{j}(t)-h_{i}(t)\right]\right)+1\right) .
$$

Hence, the greater the harvest rate $h_{j}$ (see below) of $v_{j}$ with respect to the harvest rate $h_{i}$ of $v_{i}$, the more likely agent $v_{i}$ is to change its strategy to the one of agent $v_{j}$. Agents only consider their current yields when formulating their next harvesting strategy. This assumption reflects boundedly rational behavior in the form of the agent's limited knowledge of their own and their neighbors' ecosystems. The parameter $\gamma$ controls the slope of the imitation probability function (Eq. 2) - i.e., for $\gamma \rightarrow \infty$ node $v_{i}$ would always imitate agent $v_{j}$ 's strategy if $h_{j}(t)>h_{i}(t)$, while for $\gamma \rightarrow 0$ the imitation probability tends to $1 / 2$ and is independent of the agents' harvest rates. Therefore, one can interpret $\gamma$ as an imitation tendency parameter. In fact, Traulsen et al. (2010) found this sigmoidal shape of imitation probability in a behavioral experiment.

- (3) For the next update, another waiting time is drawn from the exponential distribution (Eq. 1) and added to the update time of node $v_{i}$.

\subsection{A stylized ecosphere}

\subsubsection{Private resource dynamics}

The ecological module of our model consists of private renewable resources each following a logistic growth function, which is chosen as one of the simplest and most commonly used models of renewable resource dynamics in a constrained environment (Brander and Taylor, 1998; Keeling, 2000; Perman et al., 2003). Additionally, a harvest rate 
$h_{i}=E_{i} s_{i}$ is subtracted from the rate of change of the resource stock $s_{i} . E_{i}$ denotes the effort of agent $v_{i}$. Thus, the dynamics of the $i$ th resource are given by

$$
\frac{\mathrm{d} s_{i}}{\mathrm{~d} t}=g_{i}\left(1-\frac{s_{i}}{C_{i}}\right) s_{i}-E_{i} s_{i}
$$

Here, $g_{i}$ denotes the growth rate and $C_{i}$ the carrying capacity of the $i$ th resource stock. The strategy $S_{i}$ of agent $v_{i}$ can either be sustainable $\left(S_{i}=1\right)$, resulting in an effort $E_{i, s}=\frac{g_{i}}{2}$, or non-sustainable $\left(S_{i}=0\right)$ with an effort $E_{i, n}=\frac{3 g_{i}}{2}$. These efforts have been chosen such that the sustainable strategy coincides with the maximum sustainable yield, whereas the non-sustainable strategy leads to the full depletion of the resource stock and, consequently, no harvest at all in the long term. Note that $E_{i, \mathrm{n}}$ and $E_{i, \mathrm{~s}}$ are symmetrically separated from the critical effort $E_{i, \mathrm{c}}=g_{i}$. The latter is defined such that, for positive efforts below $E_{i, \mathrm{c}}$, the resource stock converges to a non-zero stationary state, whereas for efforts above $E_{i, \mathrm{c}}$ the resource stock collapses and converges to zero. When in interplay with the social update process, Eq. (3) is used as its analytically derived definite integral, which circumvents the need for any numerical integration methods.

\subsubsection{Resource heterogeneity}

Heterogeneous access to resources is operationalized by randomly distributing the resource capacities $C_{i}$ according to a prescribed probability density function. For this purpose, we examine the lognormal distribution

$\ln \mathcal{N}(C ; \mu, \sigma)=\frac{1}{C \sigma \sqrt{2 \pi}} \exp \left[-\frac{(\ln C-\mu)^{2}}{2 \sigma^{2}}\right], \quad C>0$,

with parameters $\mu$ and $\sigma$ (not to be confused with the standard deviation of $C$ ). It is derived from the normal distribution: a positive random variable is lognormally distributed if its logarithm is normally distributed. The lognormal distribution is therefore applicable for positive valued quantities and has a heavy tail. $\sigma$ and $\mu$ are the standard deviation and the mean of the $\log$ arithmic variable $\ln C$, respectively. The lognormal distribution occurs in variables from many fields, including biological and economic attributes (Sachs, 1984).

Figure 2 shows exemplary empirical distributions of three different types of resources to illustrate that real-world resource data can be qualitatively described by a lognormal distribution with least-squares fits revealing different $\sigma$ parameters: (i) forested land area per country $\sigma=3.83$ for the year 1991; (ii) biocapacity per country $\sigma=1.42$ computed from the Ecological Footprint Network (Ewing et al., 2008), representing the capacity of ecosystems to regenerate what people extract; and (iii) total renewable water resources data $\sigma=1.98$ characterizing the maximum yearly amount of water available to each country for the year 2012. Although the agreement between the lognormal distribution and the data

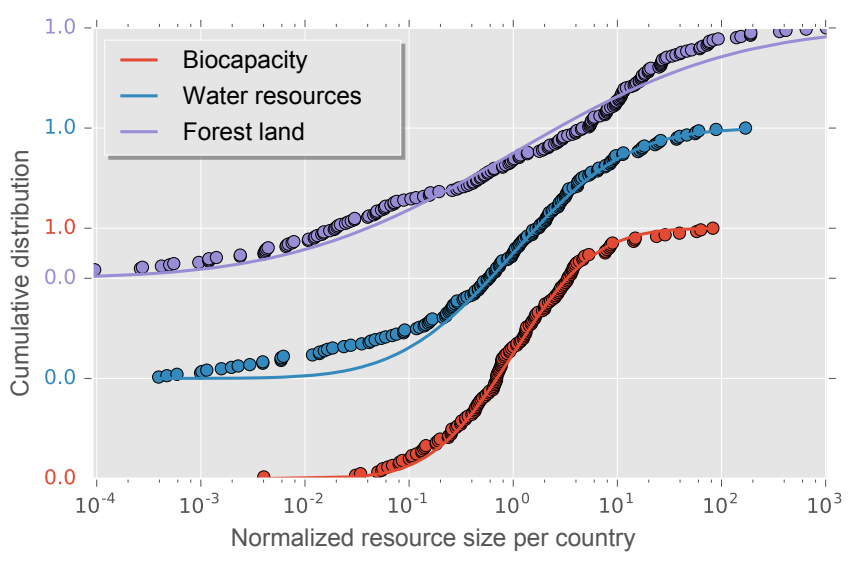

Figure 2. Empirical resource data per country normalized to the respective average (dots) together with least-squares-fitted lognormal distributions (lines): biocapacity ( $\sigma=1.42$, for the year 2007) computed from the Ecological Footprint Network (Ewing et al., 2008) represents the capacity of ecosystems to regenerate what people demand from them; total renewable water resources $(\sigma=1.98$, for the year 2012) corresponds to the maximum theoretical yearly amount of water actually available for a country; forest land area per country $(\sigma=3.83$, for the year 1991). The data are normalized to yield the same parameter $\mu=0$ of the lognormal distribution and are shifted along the $y$ axis for the sake of visibility. Note that the data qualitatively fit the lognormal distribution and that they give different values for the $\sigma$ parameters of the lognormal distribution.

is far from perfect, Fig. 2 supports the use of a lognormal model for resource heterogeneity in modeling our stylized social-ecological system.

We utilize this distribution to investigate how resource heterogeneity affects the behavior of the model in comparison to the frequently studied homogeneous case. We systematically increase parameter $\sigma$ of the lognormal distribution, which can be interpreted as a resource heterogeneity parameter, and study the resulting behavior of the model. This is done while keeping the mean of $C$ and, consequently, the cumulative carrying capacity of all resource stocks constant - i.e., the parameter $\mu$ was adjusted according to $\mu(\sigma)=-\sigma^{2} / 2$, resulting in a fixed value of one for the mean of $C$. Hence, we only ask for the effect of different resource distributions and keep the total amount of available resource stock constant.

For comparison we also present results for non-heavytailed resource capacities

$$
\begin{array}{r}
C=\left|C^{\mathrm{tmp}}\right|, \quad \text { where } \quad C^{\mathrm{tmp}} \sim \mathcal{N}\left(C^{\mathrm{tmp}} ; \mu_{\mathcal{N}}, \sigma_{\mathcal{N}}\right) \\
=\frac{1}{\sigma_{\mathcal{N}} \sqrt{2 \pi}} \exp \left[-\frac{\left(C^{\mathrm{tmp}}-\mu_{\mathcal{N}}\right)^{2}}{2 \sigma_{\mathcal{N}}^{2}}\right],
\end{array}
$$

where $\mu_{\mathcal{N}}$ now denotes the mean and $\sigma_{\mathcal{N}}$ the standard deviation of the underlying normal distribution. We also keep the mean fixed $\left(\mu_{\mathcal{N}}=1\right)$ and systematically increase the resource heterogeneity $\sigma_{\mathcal{N}}$ on comparable ranges of variances for both - normal and lognormal - distributions. Since the 
normal distribution is not bounded to positive values, we use the absolute value of the drawn random variable as the resource's carrying capacity $C$.

\subsection{Model parameterization and simulation protocol}

A model run starts with an initial condition of stocks $s_{i}(0)$ uniformly distributed between 0 and $C_{i}$ and harvesting strategies $S_{i}(0)$ drawn with a probability of 0.5 for a sustainable strategy $S_{i}=1$ or a non-sustainable strategy $S_{i}=0$. From the initial conditions, the model will converge to the steady state at $t_{f}$, where no further updates of strategy can occur. This is the case because the social network will consist solely of disconnected components with only one harvesting strategy (including the case of one single component) (Wiedermann et al., 2015). The remaining model parameters are the number of nodes $N=500$, mean degree $\bar{k}=20$, imitation tendency $\gamma=1$, and ecological growth rate $g_{i}=1$ for $i=1, \ldots N$, which are kept fixed throughout the analysis. To account for the stochasticity inherent in the model, we perform $R=250$ runs for each parameter setting of interest. We are interested in the fraction of sustainable harvesting nodes at the steady state,

$$
\left\langle S\left(t_{f}\right)\right\rangle_{N, R}=\left\langle\frac{1}{N} \sum_{i=1}^{N} S_{i}\left(t_{f}\right)\right\rangle_{R},
$$

averaged over all ensemble runs $R .\left\langle S\left(t_{f}\right)\right\rangle_{N, R}$ is bounded between one and zero, where $\left\langle S\left(t_{f}\right)\right\rangle_{N, R}=1(0)$ denotes a completely (non-)sustainable regime.

\section{Results and discussion}

\subsection{Social interaction timescale-homophily parameter space}

First, we study how the fraction of sustainable harvesting nodes at the steady state $\left\langle S\left(t_{f}\right)\right\rangle_{N, R}$ (Eq. 6) behaves in the parameter subspace spanned by the rewiring probability $\phi$ (as a measure of the degree of homophily) and the average social interaction timescale $\tau$ for vanishing resource heterogeneity $(\sigma=0.01)$ (Fig. 3a).

Four qualitatively different regimes can be observed: the sustainable regime in blue, the non-sustainable or collapse regime in red, and the transition regime in white between these, as well as, for sufficiently large $\phi$, the network fragmentation regime. The latter occurs since for large $\phi$, social dynamics are dominated by homophily and, hence, by the process of social network rewiring, and thus negligibly few changes in strategy occur. The steady state is reached by a fragmentation of the network into at least one purely sustainable and at least one purely non-sustainable component of comparable size.

In turn, for smaller $\phi$ the effect of homophily is sufficiently weak such that most agents remain connected to a single component in the social network. The steady state is reached with a large connected network component. Here, large interaction timescales $\tau$ lead to a sustainable regime. This is because the comparisons of harvest rates typically happen when the logistic resource has been harvested for a sufficiently long time to reveal that the harvest rate converges to a positive value for a sustainable strategy, whereas for a non-sustainable strategy it converges to zero.

Our main focus lies on the emergent properties of our model from a complex system's perspective. Hence, we do not claim that any quantitative choice of parameters is based on real-world assumptions. Rather, we focus here on qualitative observations in terms of general parameter regimes which in correspondence with the arbitrarily chosen ecological timescale cause a certain differential outcome of the model. However, in order to qualitatively compare our model with some real-world observations, we first look at the timescale of social updates $\tau$. It has been suggested than modern lifestyles are dominated by a social acceleration (Rosa, 2013). Simultaneously, the pressure humanity is putting on the planet (Steffen et al., 2004) has experienced a great acceleration (Steffen et al., 2015a). This can be interpreted such that faster social timescales $\tau$ lead to a non-sustainable regime, as observed in our model (see Fig. 3). Viewed with caution, the mechanisms in our model might be a possible explanation of this phenomenon. In any case, it highlights the importance of well-interacting social timescales with ecological ones. Since ecological timescales (e.g., the seasonal cycle) are difficult to influence, this suggests to take social timescales (e.g., election cycles, fashion trends, product launches) into account for possible policy interventions. Therefore, it might be worthwhile to study the relationship between social and ecological timescales more intensively to identify suitable policy actions for the benefit of a sustainable system.

We furthermore observe a linear relationship between critical parameters $\phi_{\mathrm{c}}$ and $\tau_{\mathrm{c}}$ where the transition between collapse and sustainable regimes occurs (Fig. 3). This result can be explained by the rate at which strategy changes happen. For $\phi=0$, the transition occurs at $1 / \tau \approx 1$, i.e., the ecological growth rate. For $\phi>0$, imitation interactions happen at a rate $(1-\phi) / \tau$ (Wiedermann et al., 2015) since the network rewires with probability $\phi$ and, hence, imitation takes place with probability $1-\phi$. Hence, the effective imitation rate $(1-\phi) / \tau$ equals approximately 1 (the ecological growth rate) in the transition regime, which explains the linear dependence between the two social parameters.

In other words, the homophily process in our model is beneficial for reaching the sustainable regime, where all agents harvest their resource gaining the maximum sustainable yield. All stochasticity and inherent shocks towards this sustainable steady state are absorbed and not affecting the final outcome. In this sense the sustainable regime can be described as resilient. This aligns with the findings of Newig et al. (2010), who (although from a different perspective) 
Average fraction of sustainable nodes at the consensus state

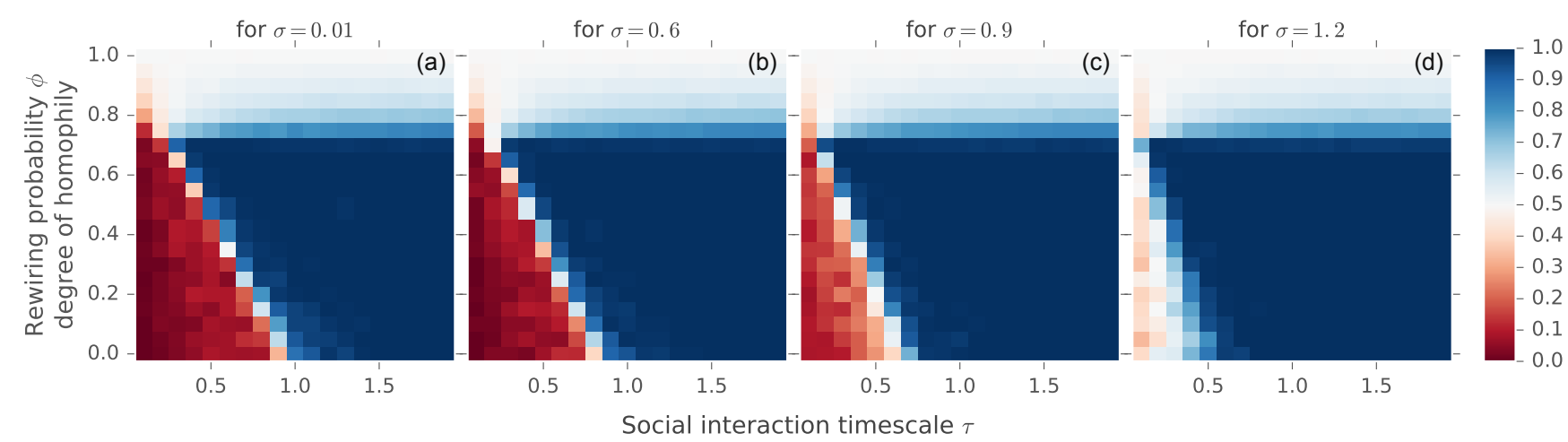

Figure 3. Social interaction timescale-homophily parameter space. Average fraction of sustainable harvesting agents in the steady state depending on the social network rewiring probability $\phi$ (measuring the degree of homophily) and the social interaction timescale $\tau$ for four distinct levels of resource heterogeneity $(\mathbf{a}: \sigma=0.01 ; \mathbf{b}: \sigma=0.6 ; \mathbf{c}: \sigma=0.9 ; \mathbf{d}: \sigma=1.2)$. One observes four qualitatively different regimes: (i) the sustainable regime for $\phi \lesssim 0.8$ and sufficiently large (slow) $\tau$ in blue, (ii) the non-sustainable or collapse regime for $\phi \lesssim 0.8$ and sufficiently small (fast) $\tau$ in red, and (iii) in between both the transition regime in white and (iv) the network fragmentation regime for $\phi \gtrsim 0.8$, also in white.

hypothesize that homophily has a beneficial effect on the resilience of a social-ecological network. Furthermore, one can interpret a large homophily parameter $\phi$ as the agents' means to protect themselves against the fast and free exchange of harvesting strategies. Along similar lines, it has been found that individuals with more environmental concerns also hold more protectionist policy preferences (Bechtel et al., 2012). Our model suggests one possible mechanism for how these relationships might come into place. However, it needs to be stated that too high a rewiring probability leads to a fragmentation of the social network into smaller groups of disjoint strategies, preventing the opportunity of a completely sustainable outcome. Thus, network adaptation at very high rates should be avoided for the sake of knowledge exchange and consensus formation.

Overall, these results demonstrate that immaterial processes distinct from macroeconomic optimization paradigms and residing exclusively in the social sphere, such as homophily and imitation, are capable of determining the eventual state of a material renewable resource. Thereby, these processes are able to govern a coupled social-ecological system such that full sustainability and total collapse are possible outcomes within the investigated social parameter space. Additionally, they show how the interaction of different social processes such as strategy imitation and homophily is able to shape the sustainable regime. This suggests that socio-cultural processes should be considered as a potentially important part of feedback loops also in more elaborate models of the "World-Earth" system.

\subsection{Systematic analysis of resource heterogeneity}

We next investigate how the transition between sustainable and non-sustainable steady states depends on the parameter $\sigma$ governing resource heterogeneity. We observe a qualitatively similar structure of parameter space for varying degrees of resource heterogeneity, but observe a decreasing extent of the non-sustainable regime for increasing $\sigma$ (Fig. 3a-d).

A more systematic analysis examines the average fraction of sustainable harvesting nodes at the consensus state $\left\langle S\left(t_{f}\right)\right\rangle_{N, R}$ for several segments of the parameter space spanned by $\tau, \phi$ and the resource heterogeneity parameters $\sigma$ $\left(\sigma_{\mathcal{N}}\right)$ - i.e., results are shown for both lognormally and normally distributed resource carrying capacities (Fig. 4). The ranges of $\sigma$ for the lognormal and $\sigma_{\mathcal{N}}$ for the normal distribution are chosen such that they correspond to comparable standard deviations.

This analysis allows for explicitly showing the effect of resource heterogeneity on the critical values $\tau_{\mathrm{c}}$ (Fig. $4 \mathrm{a}, \mathrm{c}$ ) and $\phi_{\mathrm{c}}$ (Fig. 4b, d), where the transition from the non-sustainable to the sustainable regime occurs. In general, the larger the $\sigma$ $\left(\sigma_{\mathcal{N}}\right)$, the smaller the $\tau_{\mathrm{c}}$ and $\phi_{\mathrm{c}}$. In other words, a sustainable steady state can be achieved for faster social interactions and smaller degrees of homophily the larger the resource heterogeneity is. The critical effective update timescale $\tau /(1-\phi) \stackrel{!}{=}$ $\tau_{\text {eff,crit }}$ decreases to faster update times. This behavior is more pronounced for the lognormal distribution (Fig. 4a, b) than for the normal one (Fig. 4c, d) and can be explained by the heavy tails of the lognormal distribution. For a sufficiently large resource heterogeneity $\sigma$ there is a sufficiently high probability that some agents will be assigned a comparably large resource capacity. Non-sustainable harvesting agents exploit their resources exponentially fast in time, whereas sustainable harvesting agents with comparably large resource capacity can retain their resource stock at a level that is still sufficiently large to convince other agents to become sustainable as well. 


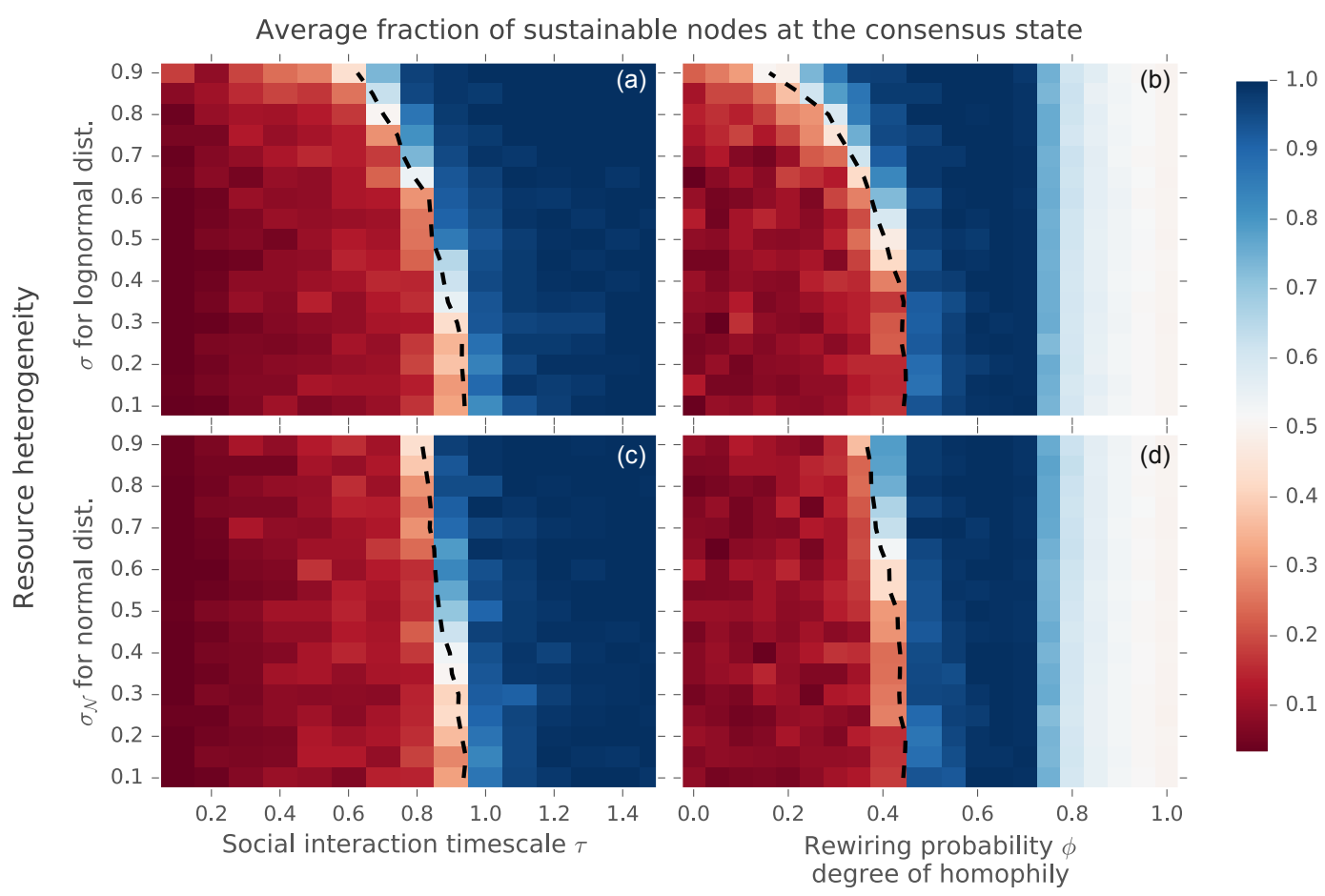

Figure 4. Effects of resource heterogeneity. Average fraction of sustainable harvesting nodes at the steady state for several segments of parameter space: (a, b) for (heavy-tailed) lognormally distributed capacities and (c, d) for (non-heavy-tailed) normally distributed capacities. Parameter spaces spanned by (a, c) social interaction timescale $\tau$ and resource heterogeneity $\sigma\left(\sigma_{\mathcal{N}}\right)$ for rewiring probability $\phi=0$, and (b, d) by $\phi$ and $\sigma\left(\sigma_{\mathcal{N}}\right)$ for $\tau=0.5$. The ranges of $\sigma$ and $\sigma_{\mathcal{N}}$ were chosen such that the standard deviations of both distributions are comparable. For both distributions, the mean was fixed to 1 . The dashed black lines indicate the linearly interpolated $50 \%$ average fraction of sustainable nodes. Note the considerable effect the lognormal resource capacity distribution (in comparison to the normal distribution) has on the critical values of $\tau$ and $\phi$, where the transition between the sustainable and the non-sustainable regime occurs.

At first, the observation that heterogeneity in access to private resources is enlarging the sustainable regime might be contradictory to reasonable assumptions. However, it demonstrates the value of a thorough system's analysis and being critical about one's own perception of what is reasonable. Cautiously comparing this phenomenon with the real world one can interpret the size of the resource capacity as the effective economic power of international macro-agents, such as world regions or nation states. This is justified, since we do not model any other economic processes but resource extraction - for example, trade, innovation and labor. The agents with comparably large economic power that employ a sustainable strategy have greater persuasive power than sustainable agents with smaller economic power. The German energy transition and its perceived impact on other countries regarding the transition towards a sustainable energy supply might be a real-world example where a country that is comparably strong economically also exerts comparably large persuasive power over other countries to move forward towards sustainable energy supply.

Overall, heterogeneity to resource access in our model demonstrates how comparably few sustainable first movers with a large resource capacity are also able to shift the over- all system toward a sustainable state at fast social interaction rates.

\section{Conclusions}

In this paper, we have studied how social-ecological thresholds between sustainable and non-sustainable resource-use regimes depend on networked social interactions (related to imitation of harvesting strategies and homophily) under conditions of resource heterogeneity. We have employed a stylized model of networked agents harvesting private renewable resources with either a sustainable or non-sustainable strategy. The strategies employed by the agents are updated through a social learning process on an adaptive social network reflecting an interconnected society. Resource heterogeneity is operationalized by lognormally and normally distributed carrying capacities of the resources.

We have shown that the properties of social processes such as strategy formation by bounded rational imitation and homophilic social network adaptation alone can precondition the long-term state of renewable resources with outcomes ranging from environmental collapse to sustainability. This observation is important because it shows that following a 
purely economic rationale may lead to neglecting decisive processes when modeling coupled social-ecological systems and suggests that more sophisticated models of global coupled human-environment systems need to consider sociocultural feedbacks as well. Furthermore, we have shown that resource heterogeneities are important model ingredients that must not be neglected, especially when resource distributions possess heavy tails. This is relevant because our findings suggest that accessible biophysical resources may indeed follow heavy-tailed distributions, and therefore the resulting resource heterogeneities may also have significant effects in more sophisticated modeling frameworks.

In the context of the ongoing debate on global change (Steffen et al., 2004) and the Anthropocene (Crutzen, 2002; Steffen et al., 2007, 2015a), such more advanced models of planetary social-ecological systems ("World-Earth" models) are needed for developing a deeper understanding of the dynamics and interrelations between planetary boundaries (Rockström et al., 2009; Steffen et al., 2015b) and social foundations (Raworth, 2012) for guiding humanity to a desirable safe and just operating space. Overall, our study highlights how socio-cultural (i.e., immaterial) dynamics and interactions can have a profound qualitative effect on physical (i.e., material) states of the environment and, consequently, that neither social processes nor resource heterogeneities should be neglected a priori in more sophisticated modeling of the "World-Earth" system.

Code availability. The code of our model (named EXPLOIT) in Cython, including a script to produce the results and related figures presented in this paper, is available at GitHub https://github. com/wbarfuss/cyexploit. For illustrative purposes, a netlogo version can be downloaded as well: https://github.com/wbarfuss/ netlogo-exploit.

Data availability. Biocapacity data were downloaded from http:// www.footprintnetwork.org/images/uploads/NFA_2010_Results.xls on 14 October 2014. Forested land area data were downloaded from http://faostat3.fao.org/download/R/RL/E on 24 November 2015. Water resources data were downloaded from http: //www.fao.org/nr/water/aquastat/data/query/index.html?lang=en on 25 November 2015.

Competing interests. The authors declare that they have no conflict of interest.

Acknowledgement. This work was developed in the context of the COPAN project on Coevolutionary Pathways in the Earth system at the Potsdam Institute for Climate Impact Research. Wolfram Barfuss is grateful for intellectual, strategic and financial support from the Heinrich Böll Foundation. Jonathan F. Donges thanks the Stordalen Foundation (via the Planetary Boundaries
Research Network PB.net), the Earth League's EarthDoc program and the Leibniz Association (project DOMINOES) for financial support. Marc Wiedermann has been supported by the German Federal Ministry for Education and Research via the BMBF Young Investigators Group CoSy-CC ${ }^{2}$ (grant no. 01LN1306A). The authors gratefully acknowledge the European Regional Development Fund (ERDF), the German Federal Ministry of Education and Research and the federal state of Brandenburg for supporting this project by providing resources on the high-performance computer system at the Potsdam Institute for Climate Impact Research. The publication of this article was funded by the Open Access Fund of the Leibniz Association. Last but not least we thank Boyan Beronov for insightful discussions and his contributions to developing a high-performance software implementation of the EXPLOIT model as well as Vera Heck for her helpful comments on the manuscript.

Edited by: S. Cornell

Reviewed by: two anonymous referees

\section{References}

Auer, S., Heitzig, J., Kornek, U., Schöll, E., and Kurths, J.: The Dynamics of Coalition Formation on Complex Networks, Scientific Reports, 5, 1-7, doi:10.1038/srep13386, 2015.

Bahar, D., Hausmann, R., and Hidalgo, C. A.: Neighbors and the evolution of the comparative advantage of nations: Evidence of international knowledge diffusion?, J. Int. Econ., 92, 111-123, 2014.

Bandura, A.: Origins of behavior, in: Social learning theory, edited by: Bandura, A., Prentice-Hall, New Jersey, USA, 15-55, 1977.

Bechtel, M. M., Bernauer, T., and Meyer, R.: The green side of protectionism: Environmental concerns and three facets of trade policy preferences, Rev. Int. Polit. Econ., 19, 837-866, 2012.

Bentley, R. A., Maddison, E. J., Ranner, P. H., Bissell, J., Caiado, C. C. S., Bhatanacharoen, P., Clark, T., Botha, M., Akinbami, F., Hollow, M., Michie, R., Huntley, B., Curtis, S. E., and Garnett, P.: Social tipping points and Earth systems dynamics, Frontiers in Environmental Science, 2, 35, doi:10.3389/fenvs.2014.00035, 2014.

Berkes, F. and Folke, C.: Linking social and ecological systems: management practices and social mechanisms for building resilience, Cambridge University Press, Cambridge, UK, 1998.

Bodin, Ö. and Tengö, M.: Disentangling intangible socialecological systems, Global Environ. Chang., 22, 430-439, 2012.

Brander, J. A. and Taylor, M. S.: The simple economics of Easter Island: A Ricardo-Malthus model of renewable resource use, Am. Econ. Rev., 88, 119-138, 1998.

Centola, D., Gonzalez-Avella, J. C., Eguiluz, V. M., and San Miguel, M.: Homophily, cultural drift, and the co-evolution of cultural groups, J. Conflict Resolut., 51, 905-929, 2007.

Chung, N. N., Chew, L. Y., and Lai, C. H.: Influence of network structure on cooperative dynamics in coupled socio-ecological systems, Europhys. Lett., 104, 28003, doi:10.1209/02955075/104/28003, 2013.

Claussen, M., Mysak, L. A., Weaver, A. J., Crucifix, M., Fichefet, T., Loutre, M.-F., Weber, S. L., Alcamo, J., Alexeev, V. A., Berger, A., Calov, R., Ganopolski, A., Goosse, H., Lohmann, G., 
Lunkeit, F., Mokhov, I. I., Petoukhov, V., Stone, P., and Wang, Z.: Earth system models of intermediate complexity: closing the gap in the spectrum of climate system models, Clim. Dynam., 18, 579-586, 2002.

Crutzen, P. J.: Geology of mankind, Nature, 415, 23-23, 2002.

Erdös, P. and Rényi, A.: On the evolution of random graphs, Publ. Math. Inst. Hungar. Acad. Sci, 5, 17-61, 1960.

Ewing, B., Goldfinger, S., Wackernagel, M., Stechbart, M., Rizk, S. M., Reed, A., and Kitzes, J.: The Ecological Footprint Atlas 2008, Global Footprint Network, Oakland, 2008.

Gross, T. and Blasius, B.: Adaptive coevolutionary networks: a review, J. R. Soc. Interface, 5, 259-271, 2008.

Hardin, G.: The tragedy of the commons. The population problem has no technical solution; it requires a fundamental extension in morality, Science, New York, NY, 162, 1243, 1968.

Holme, P. and Newman, M. E.: Nonequilibrium phase transition in the coevolution of networks and opinions, Phys. Rev. E, 74, 056108, doi:10.1103/PhysRevE.74.056108, 2006.

Janssen, M. A., Bodin, Ö., Anderies, J. M., Elmqvist, T., Ernstson, H., McAllister, R. R., Olsson, P., and Ryan, P.: Toward a network perspective of the study of resilience in social-ecological systems, Ecol. Soc., 11, 15, http://www.ecologyandsociety.org/ vol11/iss 1/art15/, 2006.

Keeling, M. J.: Multiplicative moments and measures of persistence in ecology, J. Theor. Biol., 205, 269-281, 2000.

Lade, S. J., Tavoni, A., Levin, S. A., and Schlüter, M.: Regime shifts in a social-ecological system, Theor. Ecol., 6, 359-372, 2013.

Lade, S. J., Örjan, B., Donges, J. F., Enfors, E., Galafassi, D., Olsson, P., Österblom, H., and Schlüter, M.: Modelling socialecological transformations: an adaptive network proposal, in review, 2017.

Malthus, T. R.: An essay on the principle of population, as it affects the future improvement of society. With remarks on the speculations of Mr. Godwin, edited by: Condorcet, M. and other writers, J. Johnson, London, 1798.

McPherson, M., Smith-Lovin, L., and Cook, J. M.: Birds of a feather: Homophily in social networks, Annu. Rev. Sociol., 27, 415-444, 2001.

Meadows, D. H., Goldsmith, E. I., and Meadows, P.: The limits to growth, vol. 381, Earth Island Limited, London, 1972.

Newig, J., Günther, D., and Pahl-Wostl, C.: Synapses in the network: learning in governance networks in the context of environmental management, Ecol. Soc., 15, 24, http://www. ecologyandsociety.org/vol15/iss4/art24/, 2010.

Ostrom, E.: Governing the commons, Cambridge University Press, Cambridge, UK, 2015.

Perman, R., Ma, Y., McGilvray, J., and Common, M.: Natural resource and environmental economics, Pearson Education, Harlow, UK, 2003.

Raworth, K.: A safe and just space for humanity: can we live within the doughnut, Oxfam Policy and Practice: Climate Change and Resilience, 8, 1-16, 2012.

Rockström, J., Steffen, W., Noone, K., Persson, A., Chapin, F. S., Lambin, E. F., Lenton, T. M., Scheffer, M., Folke, C., Schellnhuber, H. J., Nykvist, B., de Wit, C. A., Hughes, T., van der Leeuw, S., Rodhe, H., Sorlin, S., Snyder, P. K., Costanza, R., Svedin, U., Falkenmark, M., Karlberg, L., Corell, R. W., Fabry, V. J., Hansen, J., Walker, B., Liverman, D., Richardson, K., Crutzen,
P., and Foley, J. A.: A safe operating space for humanity, Nature, 461, 472-475, 2009.

Rosa, H.: Social acceleration: A new theory of modernity, Columbia University Press, New York, 2013.

Sachs, L.: Applied Statistics, Springer, New York, Berlin, Heidelberg, Tokyo, 1984.

Sayama, H., Pestov, I., Schmidt, J., Bush, B. J., Wong, C., Yamanoi, J., and Gross, T.: Modeling complex systems with adaptive networks, Comput. Math. Appl., 65, 1645-1664, 2013.

Scheffer, M., Carpenter, S., Foley, J. A., Folke, C., and Walker, B.: Catastrophic shifts in ecosystems, Nature, 413, 591-596, 2001.

Schellnhuber, H.-J.: Discourse: Earth System analysis - The scope of the challenge, in: Earth System Analysis, edited by: Schellnhuber, H.-J. and Wenzel, V., Springer, Berlin, 3-195, 1998.

Schellnhuber, H.-J.: Earth system analysis and the second Copernican revolution, Nature, 402, C19-C23, 1999.

Schellnhuber, H. J.: Tipping elements in the Earth System, P. Natl. Acad. Sci. USA, 106, 20561-20563, 2009.

Schleussner, C.-F., Donges, J. F., Engemann, D. A., and Levermann, A.: Clustered marginalization of minorities during social transitions induced by co-evolution of behaviour and network structure, Scientific Reports, 6, 30790, doi:10.1038/srep30790, 2016.

Snijders, T. A., Van de Bunt, G. G., and Steglich, C. E.: Introduction to stochastic actor-based models for network dynamics, Soc. Networks, 32, 44-60, 2010.

Steffen, W., Sanderson, A., Tyson, P. D., Jäger, J., Matson, P. A., Moore III, B., Oldfield, F., Richardson, K., Schellnhuber, H. J., Turner II, B. L., and Wasson, R. J.: Global Change and the Earth System: A Planet Under Pressure, Springer, Berlin, 2004.

Steffen, W., Crutzen, P. J., and McNeill, J. R.: The Anthropocene: are humans now overwhelming the great forces of nature, Ambio, 36, 614-621, 2007.

Steffen, W., Broadgate, W., Deutsch, L., Gaffney, O., and Ludwig, C.: The trajectory of the Anthropocene: The Great Acceleration, The Anthropocene Review, 2, 81-98, 2015a.

Steffen, W., Richardson, K., Rockström, J., Cornell, S. E., Fetzer, I., Bennett, E. M., Biggs, R., Carpenter, S. R., de Vries, W., de Wit, C. A., et al.: Planetary boundaries: Guiding human development on a changing planet, Science, 347, 1259855, doi:10.1126/science.1259855, $2015 \mathrm{~b}$.

Sugiarto, H. S., Chung, N. N., Lai, C. H., and Chew, L. Y.: Socioecological regime shifts in the setting of complex social interactions, Phys. Rev. E, 91, 062804, doi:10.1103/PhysRevE.91.062804, 2015.

Tavoni, A., Schlüter, M., and Levin, S.: The survival of the conformist: social pressure and renewable resource management, J. Theor. Biol., 299, 152-161, 2012.

Traulsen, A., Semmann, D., Sommerfeld, R. D., Krambeck, H.J., and Milinski, M.: Human strategy updating in evolutionary games, P. Natl. Acad. Sci. USA, 107, 2962-2966, 2010.

van Vuuren, D. P., Bayer, L. B., Chuwah, C., Ganzeveld, L., Hazeleger, W., van den Hurk, B., van Noije, T., O’Neill, B., and Strengers, B. J.: A comprehensive view on climate change: coupling of earth system and integrated assessment models, Environ. Res. Lett., 7, 024012, doi:10.1088/1748-9326/7/2/024012, 2012. van Vuuren, D. P., Lucas, P. L., Häyhä, T., Cornell, S. E., and Stafford-Smith, M.: Horses for courses: analytical tools to ex- 
plore planetary boundaries, Earth Syst. Dynam., 7, 267-279, doi:10.5194/esd-7-267-2016, 2016.

Verburg, P. H., Dearing, J. A., Dyke, J. G., van der Leeuw, S., Seitzinger, S., Steffen, W., and Syvitski, J.: Methods and approaches to modelling the Anthropocene, Global Environ. Chang., 39, 328-340, doi:10.1016/j.gloenvcha.2015.08.007, 2016.
Wiedermann, M., Donges, J. F., Heitzig, J., Lucht, W., and Kurths, J.: Macroscopic description of complex adaptive networks coevolving with dynamic node states, Phys. Rev. E, 91, 052801, doi:10.1103/PhysRevE.91.052801, 2015.

Zanette, D. H. and Gil, S.: Opinion spreading and agent segregation on evolving networks, Physica D, 224, 156-165, 2006. 